\title{
Instabilities in tensorial nonlocal gravity
}

\author{
Henrik Nersisyan, ${ }^{1, *}$ Yashar Akrami, ${ }^{1,2, \dagger}$ Luca Amendola, ${ }^{1, \ddagger}$ Tomi S. Koivisto, ${ }^{3, \S}$ Javier Rubio, ${ }^{1, \|}$ and Adam R. Solomon ${ }^{4, \|}$ \\ ${ }^{1}$ Institut für Theoretische Physik, Ruprecht-Karls-Universität Heidelberg, \\ Philosophenweg 16, 69120 Heidelberg, Germany \\ ${ }^{2}$ Institute Lorentz, Leiden University, PO Box 9506, Leiden 2300 RA, Netherlands \\ ${ }^{3}$ Nordita, KTH Royal Institute of Technology and Stockholm University, \\ Roslagstullsbacken 23, 10691 Stockholm, Sweden \\ ${ }^{4}$ Center for Particle Cosmology, Department of Physics and Astronomy, University of Pennsylvania, \\ Philadelphia, Pennsylvania 19104, USA \\ (Received 7 November 2016; published 27 February 2017)
}

\begin{abstract}
We discuss the cosmological implications of nonlocal modifications of general relativity containing tensorial structures. Assuming the presence of standard radiation- and matter-dominated eras, we show that, except in very particular cases, the nonlocal terms contribute a rapidly growing energy density. These models therefore generically do not have a stable cosmological evolution.
\end{abstract}

DOI: 10.1103/PhysRevD.95.043539

\section{INTRODUCTION}

Most extensions of general relativity are manifestly local. A priori, however, we need not impose this restriction. Like general relativity itself, most proposed theories of modified gravity are nonrenormalizable, which is often a sign of new physics at high energies. From a local high-energy theory, nonlocalities often appear in the effective theory describing low-energy physics. For example, nonlocalities appear generically when massless or light degrees of freedom are integrated out of a local fundamental theory [1-4].

Nonlocal modifications of general relativity constructed out of inverse differential operators give rise to infrared effects that become relevant at large temporal and spatial scales. The consequences of these nonlocalities are farreaching and could provide a dynamical explanation for dark energy. Numerous examples of this line of thinking can be found in the literature [5-11]. Most of the existing nonlocal gravity models are purely phenomenological and are constructed out of nonlocal operators involving the Ricci scalar only for reasons of simplicity [12-15]. It is still an open question whether we should expect these particular nonlocal structures, as opposed to something more complicated, to arise in the low-energy limit of fundamental theories. Tensorial extensions involving elements such as the Ricci or the Riemann tensors should not be a priori excluded.

Adding nonlocal interactions can also improve some of general relativity's more undesirable properties, and these seem to specifically require tensorial nonlocalities. For example, in order to alleviate the ultraviolet divergences of

\footnotetext{
*h.nersisyan@thphys.uni-heidelberg.de

†akrami@lorentz.leidenuniv.nl

*1.amendola@thphys.uni-heidelberg.de

\$tomi.koivisto@nordita.org

.j.rubio@thphys.uni-heidelberg.de

adamsol@physics.upenn.edu
}

general relativity, one has to modify the graviton propagator, which requires a tensorial term in the action [16]. Considerable recent progress has been made in ghost-free ultraviolet nonlocal gravity [17,18]. Furthermore, nonlocal modifications of general relativity could degravitate a large cosmological constant, providing an appealing solution to the problem of why a large vacuum energy does not gravitate [8]. For the purposes of degravitation, it is likely insufficient to rely on scalar degrees of freedom introduced via nonlocal scalar curvature terms. Tensorial nonlocalities, by contrast, could help implement a consistent degravitation mechanism, as is the case in the framework of massive gravity where nonlocalities modify the tensor propagator $[10,19]$.

The cosmological consequences of tensorial nonlocalities involving inverse powers of the d'Alembertian operator were considered in Refs. [20-22]. Tensor nonlocalities in these models were shown to contain rapidly growing modes, leading to instabilities in the background expansion. ${ }^{1}$ Note, however, that the inverse d'Alembertian operators considered in these references are certainly not the most general possibility that can be implemented at each order in curvature. It is possible that other wellmotivated differential operators might lead to a somewhat different evolution that is consistent with observations.

In this work we extend the analysis of Refs. [20,21] to general nonlocal tensorial actions at quadratic order in the curvature invariants and investigate whether these

\footnotetext{
${ }^{1}$ Note that these instabilities are not directly related to unitarity violation. This can be seen by considering a nonlocal term of the form $G_{\alpha \beta}\left(m^{2} / \square^{2}\right) R^{\alpha \beta}$, which results in a massive graviton propagator of a unitary form (cf. Ref. [9], where this model corresponds to $\alpha=0$ and is shown to be unitary.). However, as the action contains, in addition to scalar terms, the tensorial term $R^{\alpha \beta}\left(m^{2} / \square^{2}\right) R_{\alpha \beta}$, it will be cosmologically unstable, as can be seen from Ref. [20] and from the following.
} 
modifications are phenomenologically viable. This paper is organized as follows. In Sec. II, we introduce our tensorial nonlocal model. The cosmological consequences of this model during radiation (RD) and matter domination (MD) are discussed in Sec. III. Finally, the conclusions are presented in Sec. IV.

\section{THE $R_{\alpha \beta} \triangle^{-1} R^{\alpha \beta}$ MODEL}

Consider the most general action quadratic in the curvature invariants [16] for some differential operator $\triangle$,

$$
\begin{aligned}
S= & \frac{M_{\mathrm{Pl}}^{2}}{2} \int \mathrm{d}^{4} x \sqrt{-g}\left(-R+R f(\triangle) R+R^{\alpha \beta} g(\triangle) R_{\alpha \beta}\right. \\
& \left.+R^{\mu \nu \alpha \beta} h(\triangle) R_{\mu \nu \alpha \beta}\right)+\int \mathrm{d}^{4} x \sqrt{-g} \mathcal{L}_{m},
\end{aligned}
$$

where $M_{\mathrm{Pl}} \equiv(8 \pi G)^{-1 / 2}$ is the reduced Planck mass and $\mathcal{L}_{m}$ is the matter Lagrangian minimally coupled to gravity. Different nonlocal theories are characterized by different choices of the operator $\triangle$ and of the functions $f, g$ and $h$. In the case $\triangle=\square$, the above action is the most general parity-invariant quadratic curvature action; see Ref. [23] for derivation of the field equations. We generalize this by allowing for more general differential operators, in particular, those with curvature dependence. Note that we would recover the results of Refs. [16,23] for the quadratic truncation of the theory.

It is well motivated to consider more general forms for the operator $\triangle$; in fact the main rationale for the usual choice $\triangle=\square$ is just simplicity. For the nonlocally modified theory to be consistent on suitable backgrounds, one may need to implement a regularization $[24,25]$. For example, Ref. [25] considered a curvature-dependent regularization of the form $(\square+\hat{P})^{-1}$ with $^{2}$

$$
\begin{aligned}
\hat{P} \equiv P_{\alpha \beta}{ }^{\mu \nu}= & a R_{\left(\alpha_{\beta)}{ }^{\nu}{ }^{\nu)}\right.}+b\left(g_{\alpha \beta} R^{\mu \nu}+g^{\mu \nu} R_{\alpha \beta}\right) \\
& +c R_{(\alpha}^{(\mu} \delta_{\beta)}^{\nu)}+d R g_{\alpha \beta} g^{\mu \nu}+e R \delta_{\alpha \beta}^{\mu \nu},
\end{aligned}
$$

and $a, b, c, d$, and $e$ arbitrary constants. For example, in the de Donder gauge the graviton kinetic operator ${ }^{3}$ would

\footnotetext{
${ }^{2}$ Here and in the following, $(\mu \nu)$ denotes symmetrization over the indices and $[\mu \nu]$ denotes the antisymmetrization.

${ }^{3}$ In an isotropic and homogeneous background, the action of this operator on a tensor reduces to the action of the scalar operator on each component of the tensor [22], suggesting that the cosmological tensorial instability might be removed by dressing the inverse d'Alembertian into its appropriate tensor representation. Strictly speaking, this would take us beyond the starting point action (1) [or, otherwise, we would consider the four indices in the representation of the $(1 / \Delta)^{\mu \nu}{ }_{\alpha \beta}$ implicitly shuffling those of the $R_{\mu \nu}$ ]. Explicit construction of such models can be considered as a topic of future study; in this article we focus on the action of a general scalar (derivative) operator $1 / \Delta$ on the (Ricci) tensor $R_{\alpha \beta}$.
}

correspond to $a=-2, \quad b=0, \quad c=2, d=1 / 3$, and $e=-4 / 3$.

In the following, we also allow the differential part of the operator to assume a more generic form, involving combinations of the curvature invariants and covariant derivatives $\nabla$ that arise in explicit loop computations. We consider simple forms for the functions $g$ and $h$,

$$
g(\triangle) \equiv \frac{\bar{M}_{1}^{2}}{6 \triangle}, \quad h(\triangle) \equiv \frac{\bar{M}_{2}^{2}}{6 \triangle},
$$

with $\bar{M}_{1}$ and $\bar{M}_{2}$ mass scales to be determined by observations. These two properties allow us to simplify the action (1) for a Friedman-Lemaitre-Robertson-Walker (FLRW) background

$$
\mathrm{d} s^{2}=H^{-2} \mathrm{~d} N^{2}-a^{2} \mathrm{~d} \mathbf{x}^{2},
$$

where $N \equiv \ln a$ is the number of $e$-folds, $a$ is the scale factor, and $H \equiv \dot{a} / a$ stands for the Hubble rate with the dot denoting derivative with respect to cosmic time. Indeed, by noticing that for an FLRW metric in four dimensions the Weyl tensor

$$
C_{\mu \nu \alpha \beta} \equiv R_{\mu \nu \alpha \beta}-\left(g_{\mu[\alpha} R_{\beta] \nu}-g_{\nu[\alpha} R_{\beta] \mu}\right)+\frac{1}{3} g_{\mu[\alpha} g_{\beta] \nu} R
$$

vanishes, and using the fact that $\triangle$ is by construction metric compatible, we can write

$$
\begin{aligned}
& C_{\mu \nu \alpha \beta} \triangle^{-1} C^{\mu \nu \alpha \beta}=0 \longrightarrow \\
& R_{\mu \nu \alpha \beta} \triangle^{-1} R^{\mu \nu \alpha \beta}=-\frac{1}{3} R \triangle^{-1} R+2 R_{\alpha \beta} \triangle^{-1} R^{\alpha \beta} .
\end{aligned}
$$

Substituting this relation into Eq. (1) we obtain the simplified action

$$
\begin{aligned}
S= & \frac{M_{\mathrm{Pl}}^{2}}{2} \int \mathrm{d}^{4} x \sqrt{-g}\left(-R+R F(\triangle) R+R^{\alpha \beta} g(\triangle) R_{\alpha \beta}\right) \\
& +\int \mathrm{d}^{4} x \sqrt{-g} \mathcal{L}_{m},
\end{aligned}
$$

where we have defined $F(\triangle) \equiv f(\triangle)-\frac{\bar{M}^{2}}{18} \triangle^{-1}$ with $\bar{M}^{2}$ being a linear combination of $\bar{M}_{1}^{2}$ and $\bar{M}_{2}^{2}$. For cosmological backgrounds, the Riemann tensor does not explicitly contribute to the background evolution ${ }^{4}$; all the dynamical information can be encoded in nonlocal terms constructed out of Ricci scalars and Ricci tensors only.

The $R F(\triangle) R$ part of Eq. (7) has been extensively studied the literature for several choices of $F(\triangle)$ and $\triangle$ $[12,15,26-28]$. In this work we concentrate on the phenomenological consequences of the tensorial structure $R^{\alpha \beta} g(\triangle) R_{\alpha \beta}$. In particular, we consider the action

\footnotetext{
${ }^{4}$ Note however that it contributes at the level of perturbations.
} 


$$
\begin{aligned}
S= & \frac{M_{\mathrm{Pl}}^{2}}{2} \int \mathrm{d}^{4} x \sqrt{-g}\left(-R+\frac{\bar{M}^{2}}{6} R_{\alpha \beta} \triangle^{-1} R^{\alpha \beta}\right) \\
& +\int \mathrm{d}^{4} x \sqrt{-g} \mathcal{L}_{m},
\end{aligned}
$$

with

$$
\begin{aligned}
\triangle \equiv & m^{4}+\alpha_{1} \square+\alpha_{2} \square^{2}+\beta_{1} R_{\alpha \beta} \nabla^{\alpha} \nabla^{\beta}+\beta_{2} R \square \\
& +\gamma\left(\nabla^{\alpha} R_{\alpha \beta}\right) \nabla^{\beta},
\end{aligned}
$$

and $\alpha_{1}, \alpha_{2}, \beta_{1}, \beta_{2}, \gamma, m$ constant parameters. Up to the $m^{4}$ term, the differential operator (9) is the most general fourthorder operator containing at least one covariant derivative acting on the function following it. This choice of operator has a special physical motivation in the celebrated conformal anomaly $[29,30]$, in which quantum effects break the conformal symmetry of massless fields coupled to gravity. In this case the trace of the energy-momentum tensor receives a nonvanishing contribution from the counterterms introduced by renormalization. The form of this contribution is highly nontrivial and depends on the particle content. In four dimensions, the effective action induced by the conformal anomaly is given by [30]

$$
\begin{aligned}
S_{\mathrm{A}}= & -\frac{1}{8} \int \mathrm{d}^{4} x \sqrt{-g}\left(E-\frac{2}{3} \square R\right) \triangle_{4}^{-1} \\
& \times\left[b^{\prime}\left(E-\frac{2}{3} \square R\right)-2 b C_{\mu \nu \alpha \beta}^{2}\right],
\end{aligned}
$$

where $E \equiv R_{\mu \nu \alpha \beta}^{2}-4 R_{\mu \nu}^{2}+R^{2}$ is the Gauss-Bonnet term, $C_{\mu \nu \alpha \beta}^{2}=R_{\mu \nu \alpha \beta}^{2}-2 R_{\mu \nu}^{2}+R^{2} / 3$ is the square of the Weyl tensor, $b$ and $b^{\prime}$ are numbers that depend on the particle content of the theory, and $\triangle_{4}$ is defined as

$$
\triangle_{4}=\square^{2}+2 R_{\alpha \beta} \nabla^{\alpha} \nabla^{\beta}-\frac{2}{3} R \square+\frac{2}{3}\left(\nabla^{\alpha} R_{\alpha \beta}\right) \nabla^{\beta} .
$$

This operator is just a particular case of the operator (9) with $m=0, \alpha_{1}=0, \alpha_{2}=1, \beta_{1}=2, \beta_{2}=-2 / 3$ and $\gamma=2 / 3{ }^{5}$

The equations of motion associated to the nonlocal action (8) can be obtained by following a standard procedure for the study of nonlocal theories. We localize the action by introducing two auxiliary fields $S_{\alpha \beta}$ and $K_{\alpha \beta}$, defined as solutions of the differential equations

$$
\triangle S_{\alpha \beta}=R_{\alpha \beta}, \quad \square S_{\alpha \beta}=K_{\alpha \beta} .
$$

After variation of our nonlocal action (8) with respect to the metric $g_{\mu \nu}$ and taking into account the identity $\delta\left(\triangle^{-1}\right)=-\triangle^{-1} \delta(\triangle) \triangle^{-1}$ (see Refs. [25,31] for details) we get the modified Einstein equations

$$
R_{\alpha \beta}-\frac{1}{2} g_{\alpha \beta} R=\frac{1}{M_{\mathrm{Pl}}^{2}}\left(T_{\alpha \beta}+T_{\alpha \beta}^{\mathrm{NL}}\right),
$$

where $T_{\alpha \beta}$ is the energy-momentum tensor associated to the matter Lagrangian $\mathcal{L}_{m}$, which is by construction covariantly conserved, $\nabla_{\alpha} T_{\beta}^{\alpha}=0$. The interaction term $T_{\alpha \beta}^{\mathrm{NL}}$ arises from the variation of the nonlocal term $R_{\alpha \beta} \triangle^{-1} R^{\alpha \beta}$ and can be naturally split into six pieces,

$$
T_{\alpha \beta}^{\mathrm{NL}}=T_{\alpha \beta}^{\mathrm{NL}(0)}+T_{\alpha \beta}^{\mathrm{NL}(1)}+T_{\alpha \beta}^{\mathrm{NL}(2)}+T_{\alpha \beta}^{\mathrm{NL}(3)}+T_{\alpha \beta}^{\mathrm{NL}(4)}+T_{\alpha \beta}^{\mathrm{NL}(5)},
$$

where we have defined

$$
\begin{aligned}
& \frac{1}{2 M^{4}} T_{\alpha \beta}^{\mathrm{NL}(0)} \equiv \frac{1}{2} R_{\mu \nu} S^{\mu \nu} g_{\alpha \beta}-2 R_{\alpha}^{\mu} S_{\mu \beta}-\square S_{\alpha \beta}-g_{\alpha \beta} \nabla_{\mu} \nabla_{\nu} S^{\mu \nu}+2 \nabla_{\mu} \nabla_{\alpha} S_{\beta}^{\mu}, \\
& \frac{1}{2 \alpha_{1} M^{4}} T_{\alpha \beta}^{\mathrm{NL}(1)} \equiv \frac{1}{2} g_{\alpha \beta} \nabla_{\sigma} S^{\mu \nu} \nabla^{\sigma} S_{\mu \nu}-\nabla_{\alpha} S^{\mu \nu} \nabla_{\beta} S_{\mu \nu}-2 S^{\mu \nu} \nabla_{\nu} \nabla_{\alpha} S_{\mu \beta}+2 S_{\alpha}^{\mu} \nabla_{\nu} \nabla_{\beta} S_{\mu}^{\nu} \\
& -2 \nabla_{\mu} S^{\mu \nu} \nabla_{\alpha} S_{\beta \nu}+2 \nabla_{\nu} S_{\alpha}^{\mu} \nabla_{\beta} S_{\mu}^{\nu}+\frac{1}{2} g_{\alpha \beta} S^{\mu \nu} \nabla_{\sigma} \nabla^{\sigma} S_{\mu \nu}, \\
& \frac{1}{2 \alpha_{2} M^{4}} T_{\alpha \beta}^{\mathrm{NL}(2)} \equiv 2 K_{\beta \nu} \nabla_{\mu} \nabla_{\alpha} S^{\mu \nu}+2 \nabla_{\alpha} S^{\mu \nu} \nabla_{\mu} K_{\beta \nu}-2 \nabla_{\mu} S^{\mu \nu} \nabla_{\alpha} K_{\beta \nu}-2 S^{\mu \nu} \nabla_{\mu} \nabla_{\alpha} K_{\beta \nu} \\
& -2 K^{\mu \nu} \nabla_{\mu} \nabla_{\alpha} S_{\beta \nu}-2 \nabla_{\alpha} S_{\beta \nu} \nabla_{\mu} K^{\mu \nu}+2 \nabla_{\mu} S_{\beta \nu} \nabla_{\alpha} K^{\mu \nu}+2 S_{\beta \nu} \nabla_{\mu} \nabla_{\alpha} K^{\mu \nu} \\
& -2 \nabla_{\alpha} S^{\mu \nu} \nabla_{\beta} K_{\mu \nu}+g_{\alpha \beta} \nabla_{\sigma} S^{\mu \nu} \nabla^{\sigma} K_{\mu \nu}+\frac{1}{2} g_{\alpha \beta} S^{\mu \nu} \square K_{\mu \nu}+\frac{1}{2} g_{\alpha \beta} K_{\mu \nu} \square S^{\mu \nu} \text {, }
\end{aligned}
$$

\footnotetext{
${ }^{5}$ Note that even though the form of the operator (9) is motivated by the form of the conformal anomaly operator $\triangle_{4}$, the action (1) considered in this paper is not of the form of the action (10).
} 


$$
\begin{aligned}
\frac{1}{2 \beta_{1} M^{4}} T_{\alpha \beta}^{\mathrm{NL}(3)} \equiv & -2 R_{\alpha \sigma} \nabla_{\mu} S^{\mu \nu} \nabla^{\sigma} S_{\beta \nu}+2 R_{\beta \sigma} \nabla_{\mu} S_{\alpha \nu} \nabla^{\sigma} S^{\mu \nu}+2 R_{\beta \sigma} S_{\alpha \nu} \nabla_{\mu} \nabla^{\sigma} S^{\mu \nu}+2 R_{\alpha \sigma} S^{\mu \nu} \nabla_{\beta} \nabla^{\sigma} S_{\mu \nu} \\
+ & \frac{1}{2} R_{\alpha \beta} \nabla_{\sigma} S^{\mu \nu} \nabla^{\sigma} S_{\mu \nu}+\frac{1}{2} R_{\alpha \beta} S^{\mu \nu} \square S_{\mu \nu}-\frac{1}{2} \nabla^{\sigma} \nabla_{\alpha}\left(S^{\mu \nu} \nabla_{\beta} \nabla_{\sigma} S_{\mu \nu}\right)-2 R_{\alpha \sigma} S^{\mu \nu} \nabla_{\mu} \nabla^{\sigma} S_{\beta \nu} \\
- & \frac{1}{2} g_{\alpha \beta} \nabla^{\sigma} \nabla^{\tau}\left(S^{\mu \nu} \nabla_{\sigma} \nabla_{\tau} S_{\mu \nu}\right)+S_{\mu \alpha} S_{\beta}^{\mu}\left(\nabla^{\sigma} \nabla^{\tau} R_{\sigma \tau}\right)-\frac{1}{2} \nabla^{\sigma} \nabla_{\beta}\left(S^{\mu \nu} \nabla_{\alpha} \nabla_{\sigma} S_{\mu \nu}\right) \\
+ & \frac{1}{2} \square\left(S^{\mu \nu} \nabla_{\alpha} \nabla_{\beta} S_{\mu \nu}\right)-R_{\alpha \sigma} \nabla_{\beta} S^{\mu \nu} \nabla^{\sigma} S_{\mu \nu}-2\left(\nabla_{\mu} R^{\mu \sigma}\right)\left(S_{\alpha}^{\nu} \nabla_{\sigma} S_{\beta \nu}\right) \\
- & 2\left(\nabla_{\mu} R_{\alpha \sigma}\right)\left(S^{\mu \nu} \nabla_{\sigma} S_{\beta \nu}\right)+2\left(\nabla_{\mu} R_{\beta \sigma}\right)\left(S_{\alpha \nu} \nabla^{\sigma} S^{\mu \nu}\right)+2\left(\nabla^{\mu} R_{\mu \sigma}\right) \nabla^{\sigma}\left(S_{\alpha \nu} S_{\beta}^{\nu}\right) \\
- & \left(\nabla^{\sigma} R_{\sigma \alpha}\right)\left(S_{\mu \nu} \nabla_{\beta} S^{\mu \nu}\right)+\frac{1}{2}\left(\nabla^{\sigma} R_{\alpha \beta}\right)\left(S_{\mu \nu} \nabla_{\sigma} S^{\mu \nu}\right), \\
\frac{1}{2 \beta_{2} M^{4}} T_{\alpha \beta}^{\mathrm{NL}(4)} \equiv & S_{\alpha}^{\nu} \square R S_{\beta \nu}-R S_{\beta}^{\nu} \square S_{\alpha \nu}+S_{\beta \nu} \nabla_{\mu} \nabla_{\alpha} R S^{\mu \nu}+\nabla_{\alpha} R S^{\mu \nu} \nabla_{\mu} S_{\beta \nu}-\nabla_{\mu} R S^{\mu \nu} \nabla_{\alpha} S_{\beta \nu} \\
& -S^{\mu \nu} R \nabla_{\mu} \nabla_{\alpha} S_{\beta \nu}-S^{\mu \nu} \nabla_{\mu} \nabla_{\alpha} R S_{\beta \nu}-\nabla_{\alpha} R S_{\beta \nu} \nabla_{\mu} S^{\mu \nu} \\
& +\nabla_{\mu} R S_{\beta \nu} \nabla_{\alpha} S^{\mu \nu}+R S_{\beta \nu} \nabla_{\mu} \nabla_{\alpha} S^{\mu \nu}-\nabla_{\beta} R S^{\mu \nu} \nabla_{\alpha} S_{\mu \nu}+R_{\alpha \beta}\left(S^{\mu \nu} \square S_{\mu \nu}\right) \\
& +\frac{1}{2} g_{\alpha \beta} \nabla_{\sigma} R S^{\mu \nu} \nabla^{\sigma} S_{\mu \nu}+\frac{1}{2} g_{\alpha \beta} R S^{\mu \nu} \square S_{\mu \nu}+g_{\alpha \beta} \square\left(S^{\mu \nu} \square S_{\mu \nu}\right)-\nabla_{\alpha} \nabla_{\beta}\left(S^{\mu \nu} \square S_{\mu \nu}\right), \\
\frac{1}{2 \gamma M^{4}} T_{\alpha \beta}^{\mathrm{NL}(5) \equiv} \equiv & \frac{1}{2} g_{\alpha \beta} \nabla_{\tau}\left(S^{\mu \nu} R^{\tau \sigma} \nabla_{\sigma} S_{\mu \nu}\right)-\frac{1}{2} \nabla_{\tau}\left(S^{\mu \nu} R_{\alpha \beta} \nabla^{\tau} S_{\mu \nu}\right) \\
& +S^{\mu \nu}\left(\nabla^{\tau} R_{\tau \alpha} \nabla_{\beta} S_{\mu \nu}\right)+S^{\mu \nu}\left(\nabla_{\beta} R_{\alpha \tau} \nabla^{\tau} S_{\mu \nu}\right) \\
& +\frac{1}{2} \nabla_{\sigma} \nabla_{\alpha} \nabla_{\beta}\left(S^{\mu \nu} \nabla^{\sigma} S_{\mu \nu}\right)-\frac{1}{2} g_{\alpha \beta} \nabla_{\sigma} \nabla_{\tau} \nabla^{\sigma}\left(S^{\mu \nu} \nabla^{\tau} S_{\mu \nu}\right)-\nabla_{\sigma}\left(S_{\alpha \nu} S_{\beta}^{\nu}\left(\nabla_{\mu} R^{\mu \sigma}\right)\right),
\end{aligned}
$$

with $M^{4} \equiv \frac{1}{12} \bar{M}^{2} M_{\mathrm{Pl}}^{2}$.

\section{III. $R_{\alpha \beta} \triangle^{-1} R^{\alpha \beta}$ COSMOLOGY}

Finding exact solutions for the complicated set of equations derived in the previous section is certainly not an easy task. In what follows, we adopt the approach of Ref. [20] and assume that the energy density contributed by nonlocal effects is subdominant, so that we have the standard radiation- and matter-dominated eras $\left(T_{\alpha \beta}^{\mathrm{NL}} \ll T_{\alpha \beta}\right)$. We investigate the stability of various regions of parameter space, defined as the presence or absence of growing modes in the energy density contributed by the nonlocal interactions.

We assume $\alpha_{1}=m=0$, which allows us to find certain analytic solutions. We have carried out a preliminary numerical study for nonvanishing values of $\alpha_{1}$ and $m$ and found that the inclusion of these parameters does not significantly modify the results presented below. A full numerical study of the parameter space is beyond the scope of this work.

\section{A. Radiation-dominated era}

During radiation domination, the Ricci scalar is 0 and the terms proportional to $R \square$ and $\left(\nabla^{\sigma} R_{\sigma \tau}\right) \nabla^{\tau}$ in Eq. (9) vanish (the latter due to the Bianchi identity). On top of that, the symmetry of the FLRW metric (4) allows us to reduce the tensor $S_{\mu \nu}$ in Eq. (12) to a simple diagonal form, $S_{\mu}^{\nu}=\operatorname{diag}\left(S_{1},-S_{2},-S_{2},-S_{2}\right)$, that depends on two (homogeneous) scalar functions $S_{1}$ and $S_{2}$. Taking into account these simplifications, the set of equations (12) can be rewritten as

$$
\begin{aligned}
& \alpha_{2} S_{+}^{(4)}-6 \alpha_{2} S_{+}^{(3)}+3 \beta_{1} S_{+}^{\prime \prime}-11 \alpha_{2} S_{+}^{\prime \prime}+\left(60 \alpha_{2}-9 \beta_{1}\right) S_{+}^{\prime} \\
& \quad+8\left(\beta_{1}-4 \alpha_{2}\right) S_{+}=\frac{4 a^{4}}{\Omega_{\mathrm{R}}^{0}}, \\
& \alpha_{2} S_{-}^{(4)}-6 \alpha_{2} S_{-}^{(3)}+\left(3 \beta_{1}+5 \alpha_{2}\right) S_{-}^{\prime \prime}+\left(12 \alpha_{2}-9 \beta_{1}\right) S_{-}^{\prime}=0
\end{aligned}
$$

where $I \equiv \mathrm{d} / \mathrm{d} N$ denotes derivatives with respect to the number of $e$-folds $N, \Omega_{\mathrm{R}}^{0}$ is the current value of the critical radiation density, and we have defined two dimensionless variables

$$
S_{+} \equiv\left(S_{1}+S_{2}\right) H_{0}^{2}, \quad S_{-} \equiv\left(S_{1}-3 S_{2}\right) H_{0}^{2},
$$

in terms of the Hubble parameter today, $H_{0}^{2}=H^{2} a^{4} / \Omega_{\mathrm{R}}^{0}$. Note that for $\alpha_{2}=0$, the fourth-order differential equations (22) and (23) reduce to second-order differential equations admitting the simple solution 


$$
\begin{gathered}
S_{+}=a^{\frac{3}{2}}\left[c_{1} \sin \left(\frac{1}{2} \sqrt{\frac{5}{3}} \ln a\right)+c_{2} \cos \left(\frac{1}{2} \sqrt{\frac{5}{3}} \ln a\right)\right] \\
+\frac{a^{4}}{5 \beta_{1} \Omega_{\mathrm{R}}^{0}}, \\
S_{-}=\frac{1}{3} \tilde{c}_{1} a^{3}+\tilde{c}_{2},
\end{gathered}
$$

where $c_{1}, c_{2}, \tilde{c}_{1}$, and $\tilde{c}_{2}$ are integration constants to be fixed by initial conditions. In the general case $\alpha_{2} \neq 0$, the solution of Eqs. (22) and (23) is

$$
\begin{aligned}
S_{+}= & a^{3 / 2}\left(c_{1} a^{-q_{-}}+c_{2} a^{q_{-}}+c_{3} a^{-q_{+}}+c_{4} a^{q_{+}}\right. \\
& \left.-\frac{a^{5 / 2}}{\Omega_{\mathrm{R}}^{0}\left(24 \alpha_{2}-5 \beta_{1}\right)}\right), \\
S_{-}= & \frac{2 a^{3 / 2-y / 2}}{3-y} \tilde{c_{1}}+\frac{2 a^{3 / 2+y / 2}}{3+y} \tilde{c_{2}}+\frac{1}{3} \tilde{c_{3}} a^{3}+\tilde{c_{4}},
\end{aligned}
$$

where

$$
\begin{aligned}
q_{\mp} & =\frac{\sqrt{49 \alpha_{2}-6 \beta_{1} \mp 2 \sqrt{\left(44 \alpha_{2}-9 \beta_{1}\right)\left(12 \alpha_{2}-\beta_{1}\right)}}}{2 \sqrt{\alpha_{2}}}, \\
y & =\frac{\sqrt{25 \alpha_{2}-12 \beta_{1}}}{\sqrt{\alpha_{2}}},
\end{aligned}
$$

and $c_{i}$ and $\tilde{c}_{i}(i=1, \ldots, 4)$ are integration constants. Note that in both cases the leading contributions in $S_{+}, S_{-}$at large values of the scale factor $a$ take the power-law forms

$$
S_{+} \approx \tilde{A} a^{A}, \quad S_{-} \approx \tilde{B} a^{B},
$$

with $A$ and $B$ being positive constants related only to the model parameters $\left\{\alpha_{2}, \beta_{1}\right\}$, and $\tilde{A}$ and $\tilde{B}$ coefficients keeping track of the integration constants $c_{i}$ and $\tilde{c}_{i}(i=1, \ldots, 4)$, i.e., keeping track of the initial conditions. Inserting these asymptotic expressions into Eq. (14) and comparing the result with the standard form $T_{\nu}^{\mu}=$ $\operatorname{diag}\left(\rho_{\mathrm{NL}},-p_{\mathrm{NL}},-p_{\mathrm{NL}},-p_{\mathrm{NL}}\right)$ for a perfect fluid, we can derive approximate expressions at the lowest order in $\Omega_{\mathrm{R}}^{0}$ for the nonlocal energy density $\rho_{\mathrm{NL}}$ and the nonlocal equation of state $w_{\mathrm{NL}} \equiv p_{\mathrm{NL}} / \rho_{\mathrm{NL}}$ during radiation domination,

$$
\begin{gathered}
\rho_{\mathrm{NL}} \approx-3 M^{4} \Omega_{\mathrm{R}}^{0}\left(\tilde{A}(A+4) a^{A-4}+\tilde{B}(B+1) a^{B-4}\right), \\
w_{\mathrm{NL}} \approx-\frac{1}{3} \frac{(A-1) \tilde{A}(A+4) a^{A-4}+\tilde{B}\left(B^{2}-1\right) a^{B-4}}{\tilde{A}(A+4) a^{A-4}+\tilde{B}(B+1) a^{B-4}} .
\end{gathered}
$$

The behavior of $w_{\mathrm{NL}}$ at large values of $a$ depends on the relation between $A$ and $B$, i.e., on the precise choice of the model parameters $\left\{\alpha_{2}, \beta_{1}\right\}$. For $B<A$, the equation of state asymptotically approaches $w_{\mathrm{NL}}=-\frac{1}{3}(A-1)$, while for $B>A$ it instead evolves towards $w_{\mathrm{NL}}=-\frac{1}{3}(B-1)$. Note that, contrary to the nonlocal energy density $\rho_{\mathrm{NL}}$, the asymptotic values of $w_{\mathrm{NL}}$ do not depend on the initial conditions.

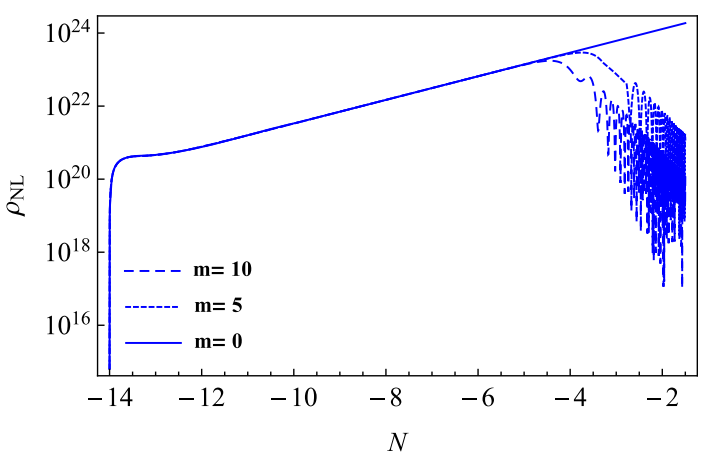

FIG. 1. Functional behavior of the nonlocal energy density $\rho_{\mathrm{NL}}$ versus the number of $e$-folds $N$ during radiation domination for an operator $\triangle=\alpha_{1} \square+m^{4}$ and different values of $m$. All quantities are expressed in units with $H_{0}=1$. Note that the dimensionful parameter $\alpha_{1}$ is not an independent parameter: together with $\bar{M}$, it fixes the amplitude of nonlocal effects and does not modify the dynamics. In this plot, we set $\bar{M}=H_{0}$ and $\alpha_{1}=H_{0}^{2}$. The late-time evolution of the nonlocal energy density develops a damped oscillatory pattern in the vicinity of $N=0$ when our radiation-domination ansatz for the scale factor $a$ is no longer applicable. The average of this quantity over an oscillation period scales as $a^{-8}$, i.e., faster than the radiation fluid $\left(\rho_{\mathrm{R}} \sim a^{-4}\right)$. A similar damping during radiation domination would require values of $m$ comparable to the Hubble rate during that era.

For $\alpha_{2}=0$ we have $A=4$ and $B=3$ [cf. Eqs. (25) and (26)]. These asymptotic values translate into a constant nonlocal energy density $\rho_{\mathrm{NL}}$ and a cosmologicalconstantlike equation of state $w_{\mathrm{NL}}=-1$. Therefore, nonlocal contributions with $\alpha_{2}=0$ can in principle lead to a viable cosmology, as long as the radiation energy density is dominant over $\rho_{\mathrm{NL}}$ for the entire radiation-dominated era. ${ }^{6}$

The situation changes completely in the $\alpha_{2} \neq 0$ case. Demanding the absence of a growing mode in Eq. (31) imposes $A, B \leq 4$. By considering Eqs. (27) and (29) with the restriction $B \leq 4$, we get the constraints

$$
\alpha_{2}>0, \quad \beta_{1} \in\left[0, \frac{25}{12} \alpha_{2}\right] \text {. }
$$

Unfortunately, these two conditions are never satisfied for $A \leq 4$. Indeed, a simple inspection of Eq. (27) shows that in order to keep $A \leq 4$ we must have $q_{+}+3 / 2 \leq 4$ and $q_{-}+3 / 2 \leq 4$, or equivalently $\beta_{1} \leq 24 / 5 \alpha_{2}$ and $\beta_{1} \geq 24 / 5 \alpha_{2}$, in clear contradiction with each other and with (33). The growing modes become rapidly dominant unless the prefactor of the nonlocal contribution in the action is largely suppressed. ${ }^{7}$

\footnotetext{
${ }^{6}$ Note that this conclusion holds only for $\alpha_{1}=0$. As shown in Ref. [20], the $\alpha_{1} \neq 0$ scenario contains growing modes and leads to an unstable cosmology.

${ }^{7}$ Note that instabilities associated with tensorial structures appear also in ultraviolet extensions of general relativity. In the case of Starobinsky inflation, the problem of instabilities coming from the tensorial components is addressed by introducing a hierarchy between energy scales of the $R^{2}$ and $R_{\mu \nu} R^{\mu \nu}$ terms [32].
} 
This conclusion does not seem to be modified for an operator $\triangle=\alpha_{1} \square+m^{4}$ with values of $m$ and $\alpha_{1}$ of order $H_{0}$ and $H_{0}^{2}$, respectively. As shown in Fig. 1 , the evolution of the nonlocal energy density in this case develops a damped oscillatory pattern in the vicinity of $N=0$, when our radiation-domination ansatz for the scale factor $a$ is no longer applicable. A similar damping during radiation domination would require values of $m$ comparable to the Hubble rate at that era.

\section{B. Matter-dominated era}

Can the instabilities generated during radiation domination be suppressed during the subsequent evolution of the Universe? To answer this question we study the behavior of a subdominant nonlocal tensorial contribution during matter domination $\left(\rho_{\mathrm{M}} \gg \rho_{\mathrm{NL}}\right)$. Taking into account the definitions in (24) (with $H_{0}^{2}=H^{2} a^{3} / \Omega_{\mathrm{M}}^{0}$ ), we can write the differential equations in (12) as

$$
\begin{aligned}
& 4 \alpha_{2} S_{+}^{(4)}-12 \alpha_{2} S_{+}^{(3)}+6 \beta_{1} S_{+}^{\prime \prime}-73 \alpha_{2} S_{+}^{\prime \prime}-12 \beta_{2} S_{+}^{\prime \prime} \\
& -3 S_{+}^{\prime}\left(9 \beta_{1}-6 \gamma-41 \alpha_{2}+6 \beta_{2}\right) \\
& +16 S_{+}\left(3 \beta_{1}+7 \alpha_{2}+6 \beta_{2}\right)=\frac{12 a^{3}}{\Omega_{\mathrm{M}}^{0}},
\end{aligned}
$$

$$
\begin{gathered}
\frac{4}{3} \alpha_{2} S_{-}^{(4)}-4 \alpha_{2} S_{-}^{(3)}-\left(3 \alpha_{2}-2 \beta_{1}+4 \beta_{2}\right) S_{-}^{\prime \prime} \\
-\left(9 \beta_{1}-6 \gamma-9 \alpha_{2}+6 \beta_{2}\right) S_{-}^{\prime}=-\frac{4 a^{3}}{\Omega_{\mathrm{M}}^{0}},
\end{gathered}
$$

with $\Omega_{\mathrm{M}}^{0}$ being the critical matter density today. As in the case of radiation domination, if we choose $\alpha_{2}=0$, then Eqs. (34) and (35) are reduced from fourth-order to secondorder differential equations. These equations can be solved analytically,

$$
S_{+}=\frac{4 a^{3}}{\Omega_{\mathrm{M}}^{0}\left(7 \beta_{1}-22 \beta_{2}+18 \gamma\right)}+c_{1} a^{p_{-}}+c_{2} a^{p_{+}},
$$

$$
S_{-}=\frac{4 a^{3}}{9 \Omega_{\mathrm{M}}^{0}\left(\beta_{1}+6 \beta_{2}-2 \gamma\right)}+\tilde{c_{1}} \frac{2\left(\beta_{1}-2 \beta_{2}\right)}{3\left(3 \beta_{1}+2 \beta_{2}-2 \gamma\right)} a^{\tilde{y}}+\tilde{c_{2}},
$$

with

$$
p_{ \pm}=\frac{9 \beta_{1}+6 \beta_{2}-6 \gamma \pm \sqrt{-47 \beta_{1}^{2}+108 \beta_{1}\left(\beta_{2}-\gamma\right)+548 \beta_{2}^{2}-72 \beta_{2} \gamma+36 \gamma^{2}}}{4\left(\beta_{1}-2 \beta_{2}\right)}, \quad \tilde{y}=\frac{3\left(3 \beta_{1}+2 \beta_{2}-2 \gamma\right)}{2\left(\beta_{1}-2 \beta_{2}\right)} \text {. }
$$

The detailed solution of Eqs. (34) and (35) for the $\alpha_{2} \neq 0$ case is cumbersome and largely irrelevant for the following discussion. On general grounds, the leading contributions to $S_{+}$and $S_{-}$at large values of the scale factor $a$ can be parametrized as ${ }^{8}$

$$
\begin{aligned}
& { }^{8} \text { Our results cover the tensorial action induced by the con- } \\
& \text { formal anomaly and the extension of the Maggiore-Mancarella } \\
& \text { model considered in Ref. [21]. For the parameters associated } \\
& \text { to the conformal anomaly }\left(\alpha_{1}=0, \alpha_{2}=1, \beta_{1}=2, \beta_{2}=-2 / 3\right. \text {, } \\
& \gamma=2 / 3) \text {, one obtains } \\
& S_{+}=a^{\frac{3}{4}}\left(c_{1} a^{-\frac{1}{4}} \sqrt{133-4 \sqrt{385}}+c_{2} a^{\frac{1}{4}} \sqrt{133-4 \sqrt{385}}+c_{3} a^{-\frac{1}{4}} \sqrt{133+4 \sqrt{385}}\right. \\
& \left.+c_{4} a^{\frac{1}{4}} \sqrt{133+4 \sqrt{385}}-\frac{2 a^{9 / 4}}{9 \Omega_{\mathrm{M}}^{0}}\right) \\
& \qquad S_{-}=2 \tilde{c_{1}} a^{\frac{1}{2}}+\frac{2}{3} \tilde{c_{2}} a^{\frac{3}{2}}+\tilde{c_{3}} a+\tilde{c_{4}}-\frac{2 a^{3}}{15 \Omega_{\mathrm{M}}^{0}},
\end{aligned}
$$

while for the case $\Delta \alpha \square^{2}\left(\alpha_{1}=\beta_{1}=\beta_{2}=\gamma=0, \quad \alpha_{2} \neq 0\right)$ considered in Ref. [21] we find

$$
\begin{aligned}
S_{+}= & a^{-\frac{1}{4}(3+\sqrt{137})}\left(c_{2} a^{\frac{\sqrt{137}}{2}}+c_{3} a^{3}+c_{4} a^{\frac{1}{2}(6+\sqrt{137})}\right. \\
& \left.+c_{1}-\frac{3 a^{\frac{1}{4}}(15+\sqrt{137})}{44 \Omega_{\mathrm{M}}^{0}}\right), \\
S_{-}= & -\frac{2}{3} \tilde{c_{1}} a^{-\frac{3}{2}}+\frac{2}{3} \tilde{c_{2}} a^{\frac{3}{2}}+\frac{\tilde{c_{3}}}{3} a^{3}+\tilde{c_{4}}-\frac{36 \ln a-44}{243 \Omega_{\mathrm{M}}^{0}} a^{3} .
\end{aligned}
$$



FIG. 2. Functional behavior of the nonlocal energy density $\rho_{\mathrm{NL}}$ versus the number of $e$-folds $N$ during matter domination for an operator $\triangle=\alpha_{1} \square+m^{4}$ and different values of $m$. All quantities are expressed in units with $H_{0}=1$. Note that the dimensionful parameter $\alpha_{1}$ is not an independent parameter. Together with $\bar{M}$ in the action, it fixes the amplitude of nonlocal effects and does not modify the dynamics. In this plot we set $\bar{M}=H_{0}$ and $\alpha_{1}=H_{0}^{2}$. The late-time evolution of the nonlocal energy density develops a damped oscillatory pattern. The average of this quantity over an oscillation period scales as $a^{-6}$, i.e., faster than the matter fluid $\left(\rho_{M} \sim a^{-3}\right)$. Note that when $m$ is of the order of the Hubble rate at matter-radiation equality, this could alleviate the previous growth during radiation domination. 
TABLE I. Characteristic values of the nonlocal equation of state $w_{\mathrm{NL}}$ during RD and MD when only one of the parameters in the nonlocal operator (9) is different from 0 . Note that the operators associated to $\beta_{2}$ and $\gamma$ vanish exactly during radiation domination. The values associated to the conformal anomaly operator (11) are also presented.

\begin{tabular}{lcc}
\hline \hline Model & $w_{\mathrm{NL}}(\mathrm{RD})$ & $w_{\mathrm{NL}}(\mathrm{MD})$ \\
\hline$\alpha_{1}$ & -1.25 & -1.45 \\
$\alpha_{2}$ & -1.79 & -2.45 \\
$\beta_{1}$ & -1 & -2 \\
$\beta_{2}$ & 0 & -1 \\
$\gamma$ & 0 & -1 \\
$m$ & $5 / 3$ & 1 \\
$\triangle_{4}$ & -1.55 & -1.92 \\
\hline \hline
\end{tabular}

$$
S_{+} \approx \tilde{C} a^{C}, \quad S_{-} \approx \tilde{D} a^{D},
$$

with the positive constants $C$ and $D$ encoding information about the model parameters, and the prefactors $\tilde{C}$ and $\tilde{D}$ tracing the initial conditions. Note that the $a^{3}$ dependence of the source term in Eqs. (34) and (35) forces $C$ and $D$ to be asymptotically larger or equal to 3 . Using Eq. (14), we can derive the nonlocal energy density

$$
\rho_{\mathrm{NL}} \approx \frac{M^{4}\left(\Omega_{\mathrm{M}}^{0}\right)^{2}}{32}\left(\tilde{E} a^{2 C-6}+\tilde{F} a^{2 D-6}\right),
$$

with $\tilde{E}$ and $\tilde{F}$ being some constants built from the free parameters of the theory and the initial conditions. Since the exponents $C$ and $D$ satisfy always the condition $C$, $D \geq 3$, we have either a constant or growing nonlocal energy density $\rho_{\mathrm{NL}}$. Therefore, the instabilities arising during radiation domination cannot be suppressed in the matter-dominated era. Note that this result also holds for the operator $\triangle=\alpha_{1} \square+m^{4}$ with nonvanishing values of $m$ and $\alpha_{1}$, with numerical results presented in Fig. 2.

For the sake of completeness, we present in Table I the asymptotic values of the nonlocal equation of state $w_{\mathrm{NL}}$ when only one of the parameters in the operator (9) is different from 0 . The values associated to the conformal anomaly operator (11) are also displayed. This helps us to see in a qualitative way the contribution coming from the different operators in (9) when the condition $T_{\alpha \beta}^{\mathrm{NL}} \ll T_{\alpha \beta}$ is satisfied. $^{9}$

\footnotetext{
${ }^{9}$ Note that this can always be achieved by fine-tuning the mass scale $\bar{M}$ in Eq. (8).
}

\section{CONCLUSIONS}

In this paper, we have explored the stability of a general class of tensorial nonlocal extensions of general relativity. Our result is a direct answer to Ref. [20], where the authors conjectured that the instabilities arising in the tensorial $R_{\alpha \beta} \square^{-1} R^{\alpha \beta}$ model might be cured by a generalization of the d'Alembertian operator to $\alpha_{1} \square+m^{4}$ or to the conformal anomaly operator $\triangle_{4}$. We have found that the growing mode and the associated instabilities of tensorial nonlocal models cannot be generically avoided by introducing the most general nonlocal operator at second order in covariant derivatives.

This conclusion holds also for a restricted version of the operator, namely $\alpha_{1} \square+m^{4}$, if the scale $m$ is chosen to be of the order of the Hubble rate today. One could alternatively consider scenarios in which $m$ is comparable to the Hubble rate at matter-radiation equality. In those cases, an oscillatory pattern arises that could be compatible with our requirement that the nonlocal contribution to the cosmic expansion be subdominant to the matter contribution. This might give rise to phenomenologically interesting features in the form of an oscillating early dark energy.

In the presence of growing modes, terms at higher and higher order in curvature are expected to become relevant, compromising the validity of the effective action (8). Although one cannot exclude the possibility of some cancellation mechanism among the various terms, a nonperturbative study within the effective nonlocal theory is quite difficult. We believe that the instabilities associated to tensorial nonlocalities should instead be addressed in the framework of local field theories by considering mechanisms able to generate well-behaved nonlocal actions in the infrared.

\section{ACKNOWLEDGMENTS}

We acknowledge support from DFG through the Project No. TRR33 "The Dark Universe." H. N. acknowledges financial support from DAAD through the program "Forschungsstipendium für Doktoranden und Nachwuchswissenschaftler." Y. A. acknowledges support from the Netherlands Organization for Scientific Research/ Dutch Ministry of Education, Culture and Science (NWO/ OCW), and also from the D-ITP consortium, a program of the NWO that is funded by the OCW. A.R.S. was supported by funds provided to the Center for Particle Cosmology by the University of Pennsylvania. 
[1] A. O. Barvinsky and G. A. Vilkovisky, Beyond the Schwinger-Dewitt technique: converting loops into trees and in-in currents, Nucl. Phys. B282, 163 (1987).

[2] A. O. Barvinsky and G. A. Vilkovisky, Covariant perturbation theory. 2: Second order in the curvature. General algorithms, Nucl. Phys. B333, 471 (1990).

[3] A. O. Barvinsky and G. A. Vilkovisky, Covariant perturbation theory. 3: Spectral representations of the third order form factors, Nucl. Phys. B333, 512 (1990).

[4] A. O. Barvinsky, Yu. V. Gusev, V. V. Zhytnikov, and G. A. Vilkovisky, Asymptotic behaviors of one loop vertices in the gravitational effective action, Classical Quantum Gravity 12, 2157 (1995).

[5] S. Deser and R. P. Woodard, Nonlocal Cosmology, Phys. Rev. Lett. 99, 111301 (2007).

[6] S. Deser and R. P. Woodard, Observational viability and stability of nonlocal cosmology, J. Cosmol. Astropart. Phys. 11 (2013) 036.

[7] R. P. Woodard, Nonlocal models of cosmic acceleration, Found. Phys. 44, 213 (2014).

[8] N. Arkani-Hamed, S. Dimopoulos, G. Dvali, and G. Gabadadze, Nonlocal modification of gravity and the cosmological constant problem, arXiv:hep-th/0209227.

[9] G. Dvali, Predictive power of strong coupling in theories with large distance modified gravity, New J. Phys. 8, 326 (2006).

[10] G. Dvali, S. Hofmann, and J. Khoury, Degravitation of the cosmological constant and graviton width, Phys. Rev. D 76, 084006 (2007).

[11] M. Maggiore, Phantom dark energy from nonlocal infrared modifications of general relativity, Phys. Rev. D 89, 043008 (2014).

[12] M. Maggiore and M. Mancarella, Nonlocal gravity and dark energy, Phys. Rev. D 90, 023005 (2014).

[13] S. Foffa, M. Maggiore, and E. Mitsou, Cosmological dynamics and dark energy from nonlocal infrared modifications of gravity, Int. J. Mod. Phys. A A29, 1450116 (2014).

[14] Y. Dirian, S. Foffa, N. Khosravi, M. Kunz, and M. Maggiore, Cosmological perturbations and structure formation in nonlocal infrared modifications of general relativity, J. Cosmol. Astropart. Phys. 06 (2014) 033.

[15] H. Nersisyan, Y. Akrami, L. Amendola, T. S. Koivisto, and J. Rubio, Dynamical analysis of $R \frac{1}{\square^{2}} R$ cosmology: Impact of initial conditions and constraints from supernovae, Phys. Rev. D 94, 043531 (2016).
[16] T. Biswas, E. Gerwick, T. Koivisto, and A. Mazumdar, Towards Singularity and Ghost-Free Theories of Gravity, Phys. Rev. Lett. 108, 031101 (2012).

[17] S. Talaganis and A. Mazumdar, High-energy scatterings in infinite-derivative field theory and ghost-free gravity, Classical Quantum Gravity 33, 145005 (2016).

[18] L. Modesto and L. Rachwal, Finite conformal quantum gravity and nonsingular spacetimes, arXiv:1605.04173.

[19] C. de Rham, S. Hofmann, J. Khoury, and A. J. Tolley, Cascading gravity and degravitation, J. Cosmol. Astropart. Phys. 02 (2008) 011.

[20] P. G. Ferreira and A. L. Maroto, A few cosmological implications of tensor nonlocalities, Phys. Rev. D 88, 123502 (2013).

[21] G. Cusin, S. Foffa, M. Maggiore, and M. Mancarella, Nonlocal gravity with a Weyl-square term, Phys. Rev. D 93, 043006 (2016).

[22] N.C. Tsamis and R. P. Woodard, A caveat on building nonlocal models of cosmology, J. Cosmol. Astropart. Phys. 09 (2014) 008.

[23] A. Conroy, T. Koivisto, A. Mazumdar, and A. Teimouri, Generalized quadratic curvature, nonlocal infrared modifications of gravity, and Newtonian potentials, Classical Quantum Gravity 32, 015024 (2015).

[24] C. Wetterich, Effective nonlocal Euclidean gravity, Gen. Relativ. Gravit. 30, 159 (1998).

[25] A. O. Barvinsky, Aspects of nonlocality in quantum field theory, quantum gravity, and cosmology, Mod. Phys. Lett. A 30, 1540003 (2015).

[26] G. Cusin, S. Foffa, M. Maggiore, and M. Mancarella, Conformal symmetry and nonlinear extensions of nonlocal gravity, Phys. Rev. D 93, 083008 (2016).

[27] C. Deffayet and R. P. Woodard, Reconstructing the distortion function for nonlocal cosmology, J. Cosmol. Astropart. Phys. 08 (2009) 023.

[28] T. Koivisto, Dynamics of nonlocal cosmology, Phys. Rev. D 77, 123513 (2008).

[29] D. M. Capper and M. J. Duff, Trace anomalies in dimensional regularization, Nuovo Cimento A 23, 173 (1974).

[30] R. J. Riegert, A nonlocal action for the trace anomaly, Phys. Lett. B 134, 56 (1984).

[31] M.E. Soussa and R. P. Woodard, A nonlocal metric formulation of MOND, Classical Quantum Gravity 20, 2737 (2003).

[32] V. F. Mukhanov and G. V. Chibisov, Quantum fluctuations and a nonsingular universe, Pis'ma Zh. Eksp. Teor. Fiz. 33, 549 (1981) [JETP Lett. 33, 532 (1981)]. 